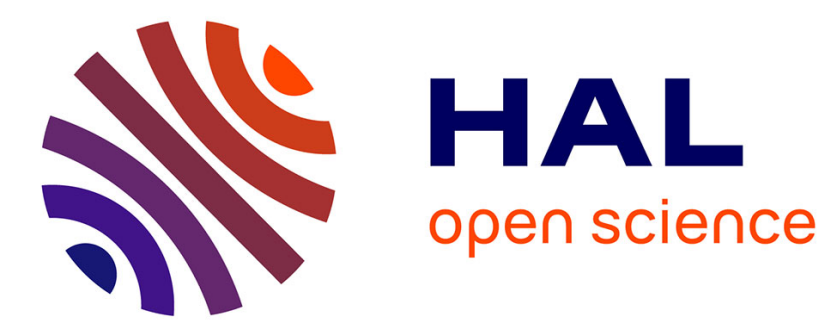

\title{
Surfaces with pulleys and Khovanov homology
}

Benjamin Audoux

\section{To cite this version:}

Benjamin Audoux. Surfaces with pulleys and Khovanov homology. Journal of Knot Theory and Its Ramifications, 2011, 20 (7), pp.1021-1040. 10.1142/S0218216511009091 . hal-01217084

\section{HAL Id: hal-01217084 \\ https://hal.science/hal-01217084}

Submitted on 20 Oct 2015

HAL is a multi-disciplinary open access archive for the deposit and dissemination of scientific research documents, whether they are published or not. The documents may come from teaching and research institutions in France or abroad, or from public or private research centers.
L'archive ouverte pluridisciplinaire HAL, est destinée au dépôt et à la diffusion de documents scientifiques de niveau recherche, publiés ou non, émanant des établissements d'enseignement et de recherche français ou étrangers, des laboratoires publics ou privés. 


\title{
SURFACES WITH PULLEYS AND KHOVANOV HOMOLOGY
}

\author{
BENJAMIN AUDOUX
}

\begin{abstract}
In this paper, we define surfaces with pulleys which are unions of 1 and 2-dimensional manifolds, glued together on a finite number of points of their interiors. Then, by seeing them as cobordisms, we give a refinment of Bar-Natan geometrical construction of Khovanov homology which can be applied to different notions of refined links as links in $I$-bundle or braid-like links.
\end{abstract}

A decade ago, M. Khovanov defined a bigraded homology categorifying the Jones polynomial for links in $S^{3}$ [Kho00]. The construction is based on the combinatorics of the different resolutions of a link diagram $D$, i.e. the possible choices for smoothing every crossing (see Fig. 1), and more precisely on how the connected components, called circles, of these resolutions behave when changing the smoothing of a crossing.

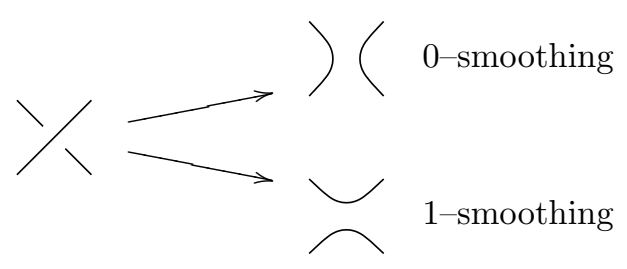

Figure 1: Smoothing for a crossing

Several refinments have been then developped, leading to triply graded homological invariants for links in I-bundle APS04, braid-like links AF05] or star-like links Aud09. In particular, the first two includes closed braids invariant. In all these examples, the main ingredient of the refinment is the splitting of the set of circles into two subsets, one of $d$-circles and one of $h$-circles.

In 2003, Bar-Natan gave a pictural construction of Khovanov homology BN05 which belongs to the realm of chain complexes over cobordisms. Bar-Natan construction can be adapted to the case of links in a $I$-bundle $Y$ (resp. braid and star-like links) by marking $h$-circles (see CS09). Actually, one can even label the circles with their values in $H_{1}(Y, \mathbb{Z} / 2 \mathbb{Z})$ (resp. $\mathbb{Z} / 2 \mathbb{Z}, 0$ standing for $d$ and 1 for $h$ ) and asking that for any cobordism, the sum over the labels of the boundary components vanishes. However, since we are dealing with abstract surfaces, this description is not very stricking as it does not figure why a non zero labelled circle should not be capped off.

In this short note, we set a general background which fits the three cases mentionned above and we give a pictural construction from which we can recover the corresponding refined Khovanov homologies.

For a given link diagram $D$, we define the set

$$
\operatorname{Res}_{D}=\bigcup_{\substack{r \text { resolution } \\ \text { of } D}}\{\text { circles of } r\}
$$

where, in general, the union is not disjoint, in the sense that a same circle appears whatever the smoothing of a crossing which is not adjacent to this circle is. If $D$ lives on an orientable surface,

Date: November 24, 2009. 
the switch of a crossing resolution splits one circle in two or merges two in one. Three different circles are then involved. We say that a map $\phi: \operatorname{Res}_{D} \longrightarrow\{d, h\}$ is a refining map if, among these three circles, there can not be exactly one send to $h$. In other words, if one of them is send to $d$ by $\phi_{D}$, then the other two have the same image through $\phi_{D}$.

A refined link diagram is a pair $\left(D, \phi_{D}\right)$ where $D$ is a link diagram on an orientable surface and $\phi_{D}: \operatorname{Res}_{D} \longrightarrow\{d, h\}$ is a refining map.

Now, suppose $\left(D_{1}, \phi_{D_{1}}\right)$ and $\left(D_{2}, \phi_{D_{2}}\right)$ are two refined link diagrams such that $D_{1}$ and $D_{2}$ are identical outside a disk where they differ by a Reidemeister move. Then there are several natural maps defined between some subsets of $\operatorname{Res}_{D_{1}}$ and $\operatorname{Res}_{D_{2}}$. We say that $\left(D_{1}, \phi_{D_{1}}\right)$ and $\left(D_{2}, \phi_{D_{2}}\right)$ are connected by a refined Reidemeister moves if $\phi_{D_{1}}$ and $\phi_{D_{2}}$ factorize through these maps and if the special circles as shown in Fig. 2 are send to $d$.

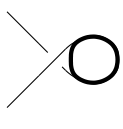

RI

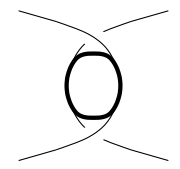

RII

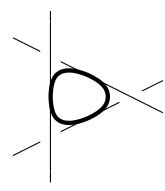

RIII

Figure 2: Special circles : for every Reidemeister, the special circle is shown in bold.

For instance, one can consider the diagrams on a surface $\Sigma$ together the map which send a circle to $d$ if it is null-homotopic and to $h$ otherwise. It is straighforward to check that it is a refining map and that two diagrams are linked by refined Reidemeister moves if and only if they represent the same link in $\Sigma \times[0,1]$. The maps sending a circle to its type as defined in [AF05 and Aud09] are also refining maps and they lead, respectively to the notion of braid and star-like links.

In section 1. we define surfaces with pulleys which are, broadly speaking and up to some relations, 1 and 2-dimensional pieces glued together on a finite number of points of their interiors. Such connecting points, called pulleys, are labelled by elements of $\mathbb{Z} / 3 \mathbb{Z}$. Since they have boundary, they can be considered as some multi-dimensional cobordisms. Then, we define the category $\mathcal{P}$ of chain complexes over surfaces with pulleys. We also define a map from a refined version of Bar-Natan category Kob to $\mathcal{P}$. This allows, in section 2, to import Bar-Natan construction to $\mathcal{P}$, defining refined Khovanov homology.

Theorem 1. The refined Khovanov homology is invariant under refined Reidemeister moves.

Finally, the note ends with several functors which send this geometrical construction to the algebraic world of modules.

Acknowledgement: The author would like to thank Michael Eisermann and Christine Lescop for interesting discussion and remarks. He is also grateful to Mucyo Karemera for his help in finding the functor $F_{0}$.

\section{Category of surfaces With pulleys}

1.1. Surfaces with pulleys. Surfaces with pulleys are abstract surfaces composed of 1 and $2-$ dimensional pieces that can be connected thanks to $\mathbb{Z} / 3 \mathbb{Z}$-labelled pulleys. The 2 -dimensional pieces are called surfaces and the 1-dimensional ones ropes. In Fig. 1.1. we give a generating set of elementary surfaces. Any two surfaces can be composed vertically by disjoint union. Moreover, if the right boundary of a surface corresponds to the left boundary of another surface, then they can be composed horizontally by gluing them along this common boundary (see Fig. 1.1). 


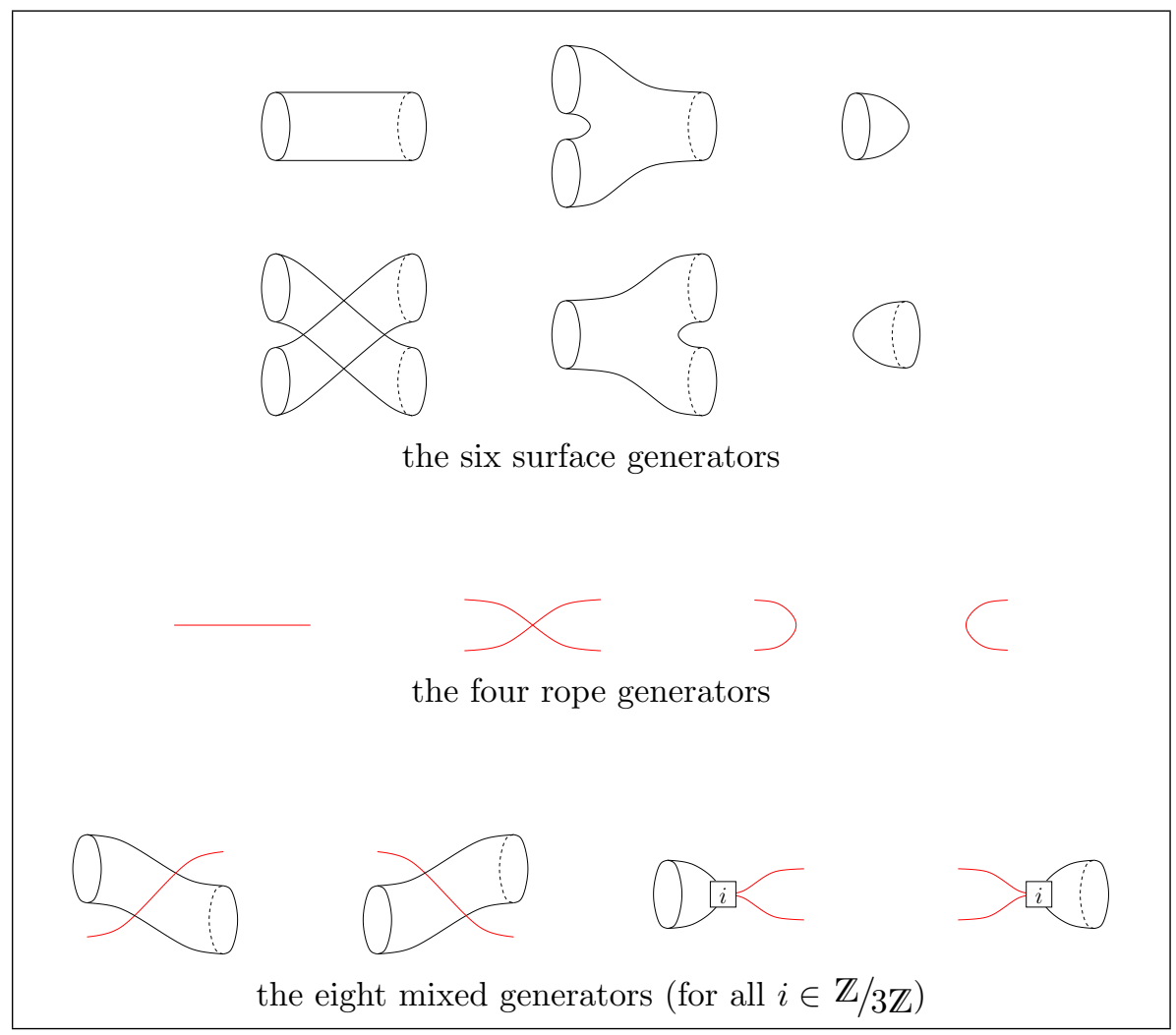

Figure 3: Generators for surfaces with pulleys

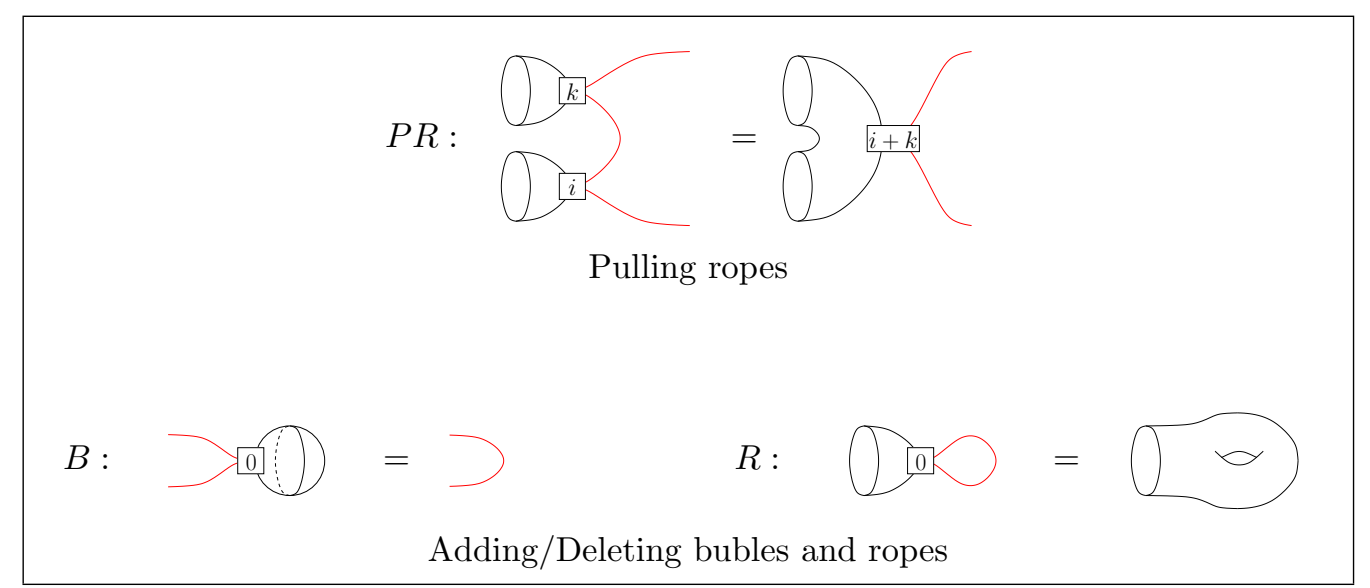

Figure 4: Special relations on surfaces with pulleys

Besides the natural relations on surfaces and ropes (see Appendix A), we quotient by some mixed relations which are given in Fig. 1.1 .

The following proposition is a direct application of relations $P R$ and $R$. 


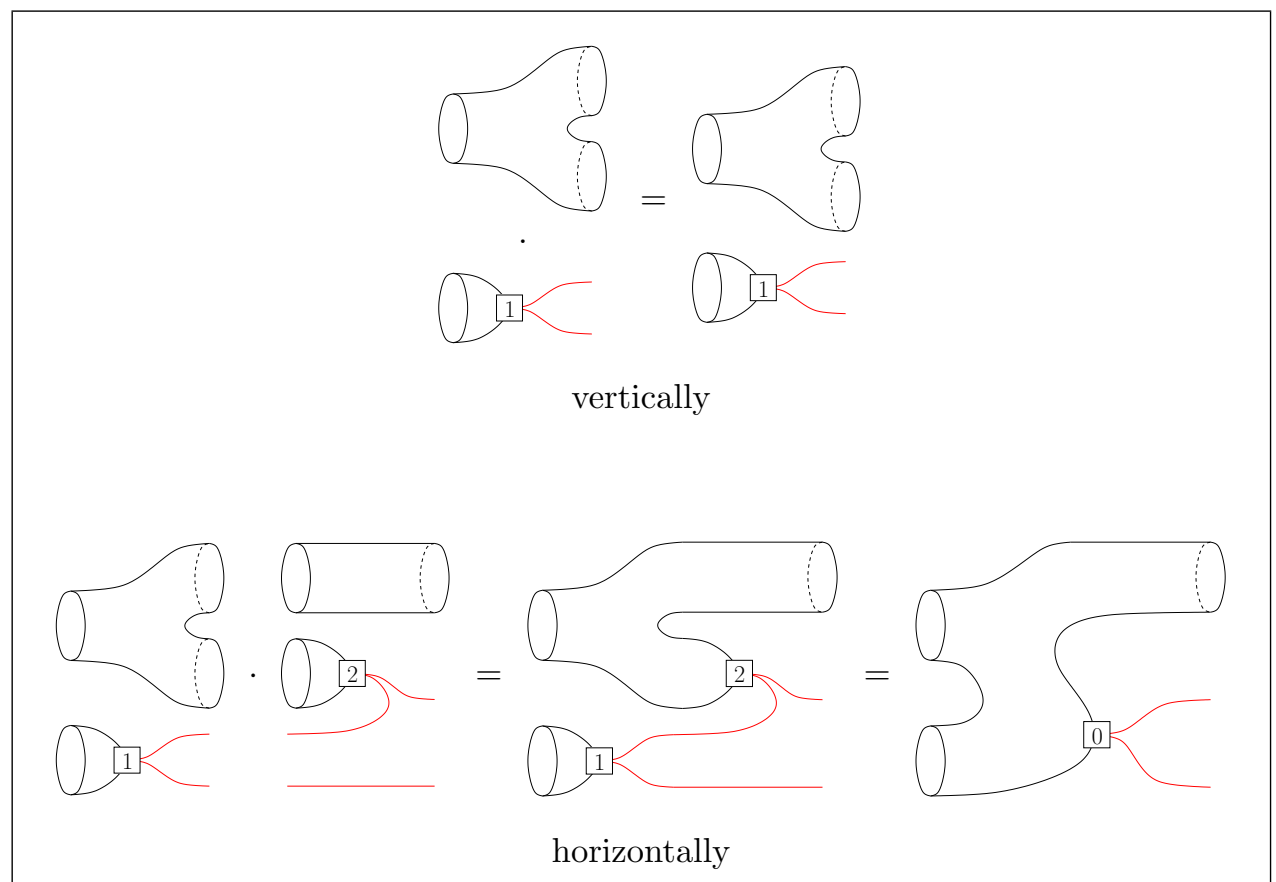

Figure 5: Composition of surfaces

Proposition 1.1. As surfaces with pulleys, we have for every $i, k \in \mathbb{Z} / 3 \mathbb{Z}$ such that $i+k=0$

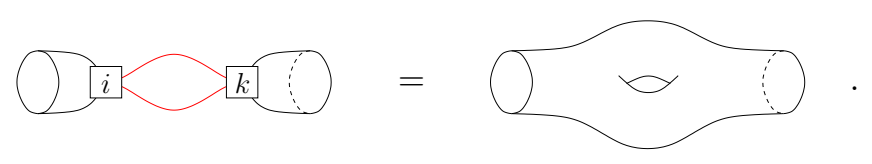

1.2. Definition of the category. We follow the definition and notation of Bar-Natan in BN05.

First, we define the category of surfaces with pulleys that we denote $\widetilde{\mathcal{P}}$. Its objects are disjoint unions of dots and circles. A morphism from $O_{1}$ to $O_{2}$ is a surfaces with pulleys $\Sigma$ whose left boundary is $O_{1}$ and right boundary $O_{2}$.

The composition of morphisms correspond to horizontal composition of surfaces with pulleys.

Now, we define $\mathcal{P}_{a b}$ which is a pre-additive category set up from $\widetilde{\mathcal{P}}$. Its objects are the same but its morphisms are formal linear combinations (possibly empty) of morphisms of $\widetilde{\mathcal{P}}$ up to the relations $S, 2 P$ and $4 T$ given in Fig. 1.2 .

Notation 1.2. We denote by $\mathcal{P}$ the category defined as $\operatorname{Kom}_{/ h}\left(\operatorname{Mat}\left(\mathcal{P}_{a b}\right)\right)$ with coefficients with coefficients in a ring $A$.

We can note that the relation $T$ is equivalent to the following relation

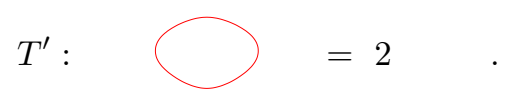

Moreover, as soon as surface with pulley does contain any 1-dimensional piece, applying $4 T$ to the pattern 03 proves that the relation $T$ is implied by others relations.

Proposition 1.3. If 2 is invertible in $A$, then any connected surface with at least two pulleys vanishes in $\mathcal{P}_{a b}$ and thus in $\mathcal{P}$. 


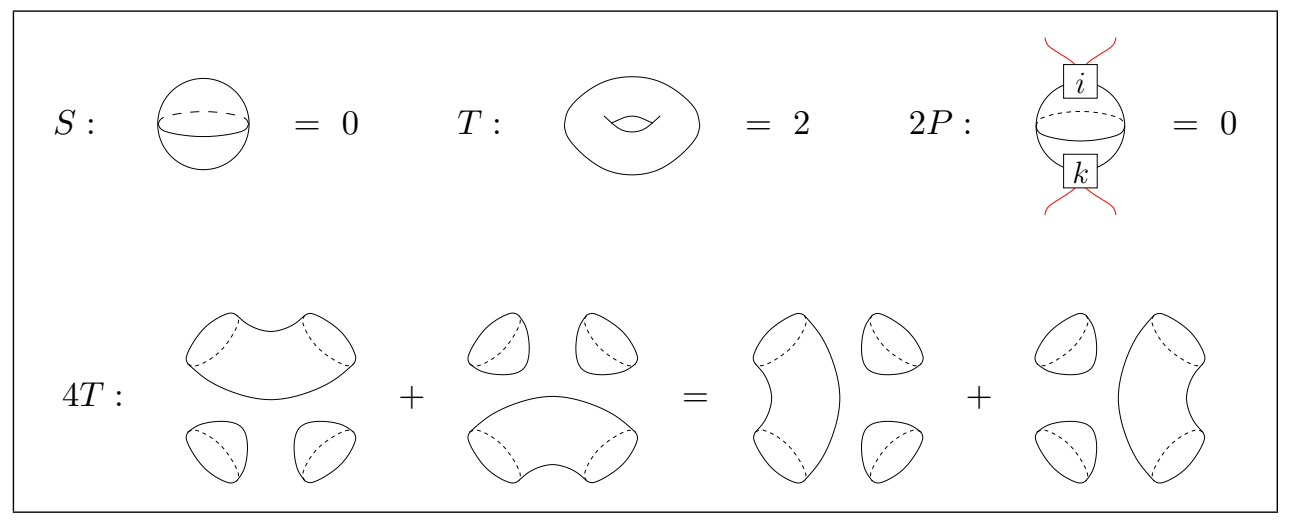

Figure 6: Relations in $\mathcal{P}_{a b}$

Proof. If 2 is invertible in $A$, then the $4 T$ relation is equivalent to the neck cutting relation (see BN05]) and thus we have

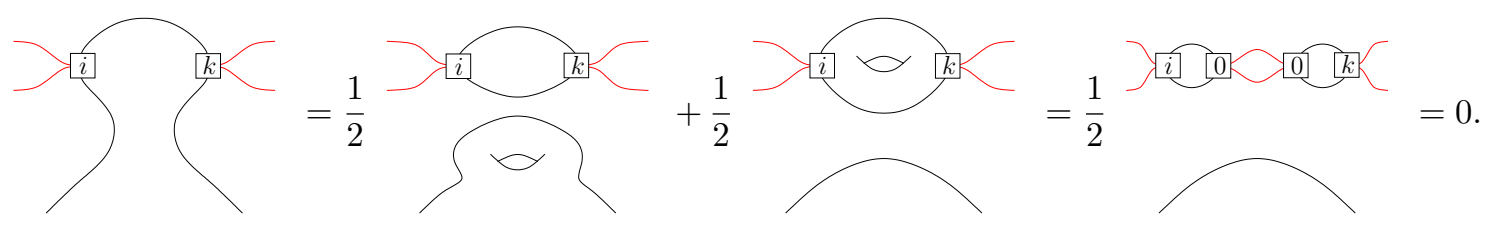

Corollary 1.4. Let $S$ be a connected surface of genus $g$ with $k$ pulleys on it. Then, if 2 is invertible in $A$ and if $g+k \geq 2$, then $S$ vanishes in $\mathcal{P}$.

1.3. A functor from refined $\mathrm{Kob}_{r}$ to $\mathcal{P}$. We consider $\mathrm{Kob}_{r}$ a refined version of the category Kob, defined as the latter but over the category $\operatorname{Cob}_{r}$ whose objects are circles labelled by $d$ or $h$ and morphisms are cobordisms which have no or more than two boundary components labelled by $h$.

Now, we define the map $\mathcal{F}$ from the category $\mathrm{Kob}_{r}$ to $\mathcal{P}$ as follows.

For an elementary object $O$ of $\mathrm{Kob}_{r}$, that is an object of $\mathrm{Cob}_{r}, \mathcal{F}(O)$ is obtained by forgetting $d$ labels and replacing $h$-labelled circles by dots.

Now, let $C$ be a connected cobordism between $O_{1}$ and $O_{2}$. We define the type of $C$ as

$$
\left(k_{1}, k_{2}\right)=\left(\#\left\{h \text {-labelled components of } O_{1}\right\}, \#\left\{h \text {-labelled components of } O_{2}\right\}\right) .
$$

If $k_{1}+k_{2}=0$ then $\mathcal{F}(C)$ is the natural embedding of $C$ into $\mathcal{P}$. If $k_{1}+k_{2}=2$ then $\mathcal{F}(C)$ is obtained by capping off the two $h$-circles and adding a pulley on $C$ labelled by $k_{2}-k_{1}$ (see Fig. 7). Otherwise $\mathcal{F}(C)=0$.

Then $\mathcal{F}$ is extended to unconnected morphisms by vertical composition and to chain complexes by linearity.

Proposition 1.5. If 2 is invertible in $A$, then $\mathcal{F}$ is a functor, i.e. for two horizontally composable morphisms $C_{1}$ and $C_{2}$, we have

$$
\mathcal{F}\left(C_{1} \cdot C_{2}\right)=\mathcal{F}\left(C_{1}\right) \cdot \mathcal{F}\left(C_{2}\right)
$$

First we prove two lemmata where 2 is assumed to be invertible in $A$.

Lemma 1.6. If $C_{1}$ or $C_{2}$ is a disjoint union of a connected cobordism of type $(0,0)$ with some cylinders, then the equation (1) holds. 


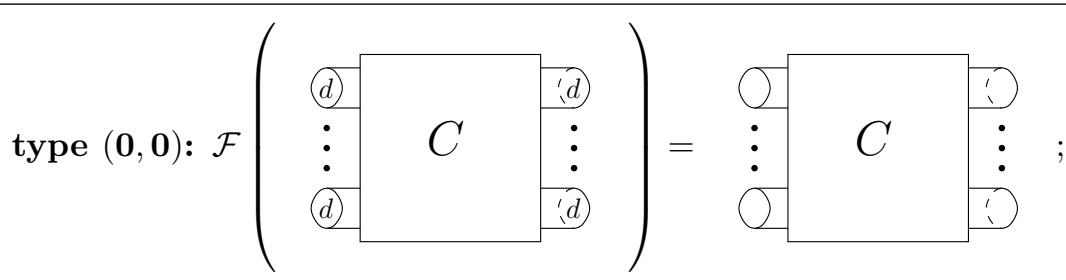

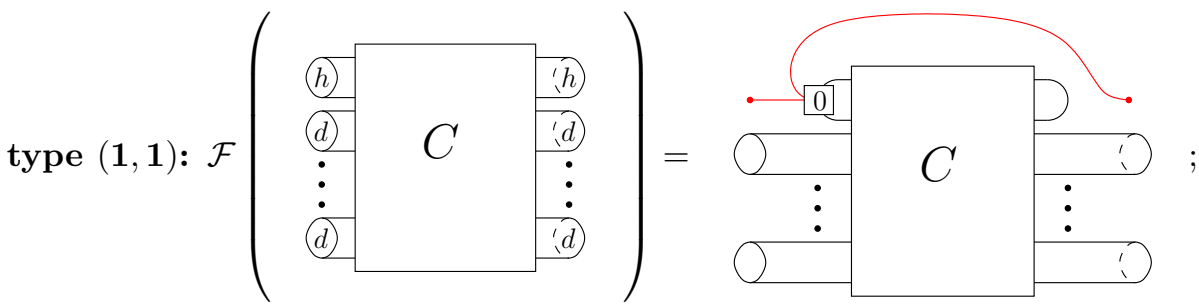

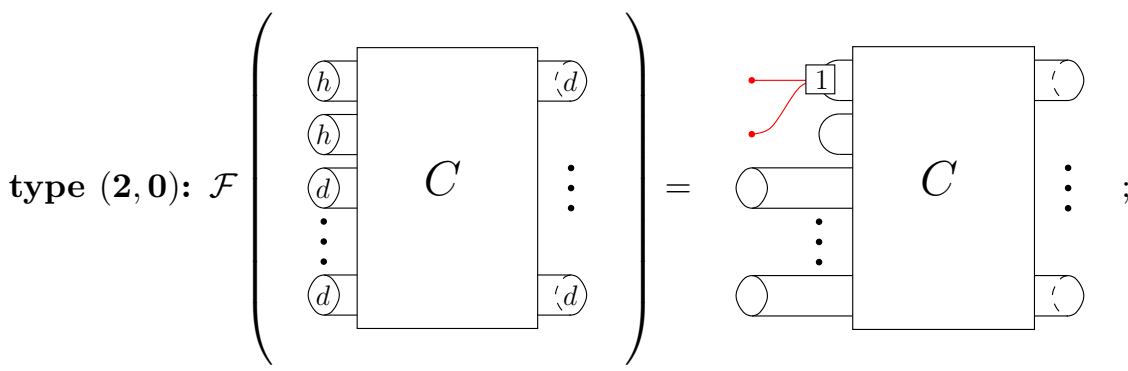

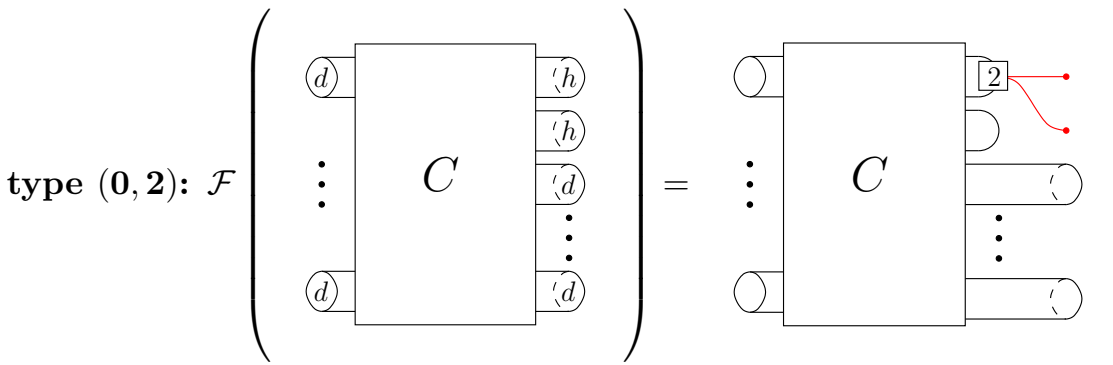

Figure 7: the functor $\mathcal{F}$

Proof. Both ends of a cylinder have necessarily the same image through $\phi$ and composing with a cylinder whose boundary components are send to $h$ corresponds to adding a bubble which can be removed thanks to the relation $B$.

Now, we assume that $C_{2}$ is the disjoint union of $D$, a connected cobordism of type $(0,0)$, with some cylinders. The only annoying case which may happen is that $D$ is connecting several cobordism of type $\left(k_{1}, k_{2}\right)$ with $k_{1}+k_{2}=2$. But then, in one hand, $\mathcal{F}\left(C_{1} \cdot C_{2}\right)$ vanishes because of its type and, in the other hand, $\mathcal{F}\left(C_{1}\right) \cdot \mathcal{F}\left(C_{2}\right)$ vanishes because of Cor. 1.4. since a connected surface have at least two pulleys on it. 
Lemma 1.7. If a corbordism $C$ can be factorized through the disjoint union of a $h$-pants, that is

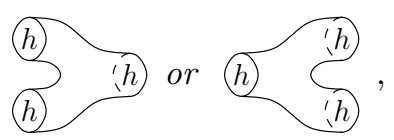

and some cylinders, then $\mathcal{F}(C)=0$.

If $C$ is a punctured sphere, then the converse is also true.

Proof. Without loose of generality, we can assume that $C$ factorizes from the left. If it factorizes through a $h$-pants of type $(2,1)$ then the connected component of the right summand connected to the $h$-pants has at least one boundary component send to $h$ and thus is of type $\left(k_{1}, k_{2}\right)$ with $k_{1}+k_{2} \geq 2$. But then, $C$ is of type $\left(1+k_{1}, k_{2}\right)$ and $\mathcal{F}(C)=0$.

Now, suppose the $h$-pants is of type $(1,2)$. Then, there are two cases :

it connects two connected components of the right summand: for the same reason than above, the two connected components are of type $\left(k_{1}^{1}, k_{2}^{1}\right)$ and $\left(k_{1}^{2}, k_{2}^{2}\right)$ with $\left(k_{1}^{1}+k_{2}^{1}\right),\left(k_{1}^{2}+\right.$ $\left.k_{2}^{2}\right) \geq 2$ and then $C$ is of type $\left(k_{1}^{1}+k_{1}^{2}-1, k_{2}^{1}+k_{2}^{2}\right)$ and $\mathcal{F}(C)=0$;

it is twice connected to a connected component of the right summand: we assume, ad absurdum, that $\mathcal{F}(C) \neq 0$ but then it is a surface of genus at least one and, because of the third boundary component of the $h$-pants, it has at least one pulley. Then it vanishes because of Cor.

In the case of a punctured sphere, the converse is clear.

Proof of Prop. 1.5. It is sufficient to prove the proposition for $C_{1}$ and $C_{2}$ two composable cobordisms such that the product $\mathcal{C}_{1} \cdot C_{2}$ is connected. The product can be decomposed as in Figure 8 where the $S_{i}^{j}$ are punctured spheres and $\Sigma$ is a disjoint union of cylinder which permutes the in and out entries. According to Lemma $1.6, \mathcal{F}\left(C_{1}\right) \cdot \mathcal{F}\left(C_{2}\right)=\mathcal{F}\left(S_{1}\right) \cdot \mathcal{F}(D) \cdot \mathcal{F}\left(S_{2}\right)$.

We prove by induction on $n_{1}+n_{2}$ that if $\mathcal{F}\left(S_{i}^{j}\right)=0$ for some $i \in\{1,2\}$ and some $j \in \llbracket 1, n_{i} \rrbracket$ then $\mathcal{F}\left(C_{1} \cdot C_{2}\right)=0$. Without loose of generality, we may assume that $i=j=1$.

If $\mathbf{n}_{\mathbf{1}}+\mathbf{n}_{\mathbf{2}} \leq \mathbf{2}: \mathcal{F}\left(S_{1}^{1}\right)=0$, thus, according to Lemma 1.7, $S_{1}^{1}$ factorizes through a $h$-pants. If it factorizes from the left, then so does $C_{1} \cdot C_{2}$ and $\mathcal{F}\left(C_{1} \cdot C_{2}\right)=0$. If it factorizes from the right, then the $h$-pants is of type $(1,2)$. Let $\left(k_{1}, k_{2}\right)$ be the type of the left summand of the factorization. Since $n_{2} \leq 2-n_{1} \leq 1$ the two right boundary components are connected to $S_{2}^{1}$, imposing that $C_{1} \cdot C_{2}$ is of genus at least 1 . Now, if $k_{1}>0$, then assuming that $\mathcal{F}\left(C_{1} \cdot C_{2}\right) \neq 0$ means that $\mathcal{F}\left(C_{1} \cdot C_{2}\right)$ contains a surface of genus greater than one with at least one pulley on it. Since Cor. 1.4 it vanishes.

If $k_{1}=0$, then $k_{2} \geq 2$, and $S_{1}^{1}$ has a third connection with $S_{2}^{1}$. Then the genus of $C_{1} \cdot C_{2}$ is at least 2 and, once again, $\mathcal{F}\left(C_{1} \cdot C_{2}\right)$ vanishes because of Cor. 1.4 .

If $\mathbf{n}_{\mathbf{1}}+\mathbf{n}_{\mathbf{2}}>\mathbf{2}$ : We follow the same discussion as above but then, there is one new possible case: $S_{1}^{1}$ may factorize from the right with a $h$-pants which connects $S_{2}^{i}$ and $S_{2}^{j}$ with distincts $i, j \in \llbracket 1, n_{2} \rrbracket$. But then, the $h$-pants may be move from $S_{1}$ to $S_{2}$ without changing $C_{1} \cdot C_{2}$ nor $\mathcal{F}\left(S_{1}\right) \cdot \mathcal{F}(D) \cdot \mathcal{F}\left(S_{2}\right)$ since in both cases, one of the summand vanishes. Moreover, the number of punctured sphere is reduced by one and one of them factorizes through a $h$-pants. We can thus apply the recursive hypothesis.

Now, we can assume that $\mathcal{F}$ does not vanish on the punctured sphere and hence that, for all $i \in\{1,2\}$ and $j \in \llbracket 1, n_{i} \rrbracket, \mathcal{F}\left(S_{i}^{j}\right)$ is of one of the following forms:

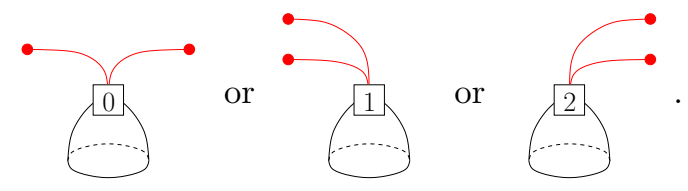




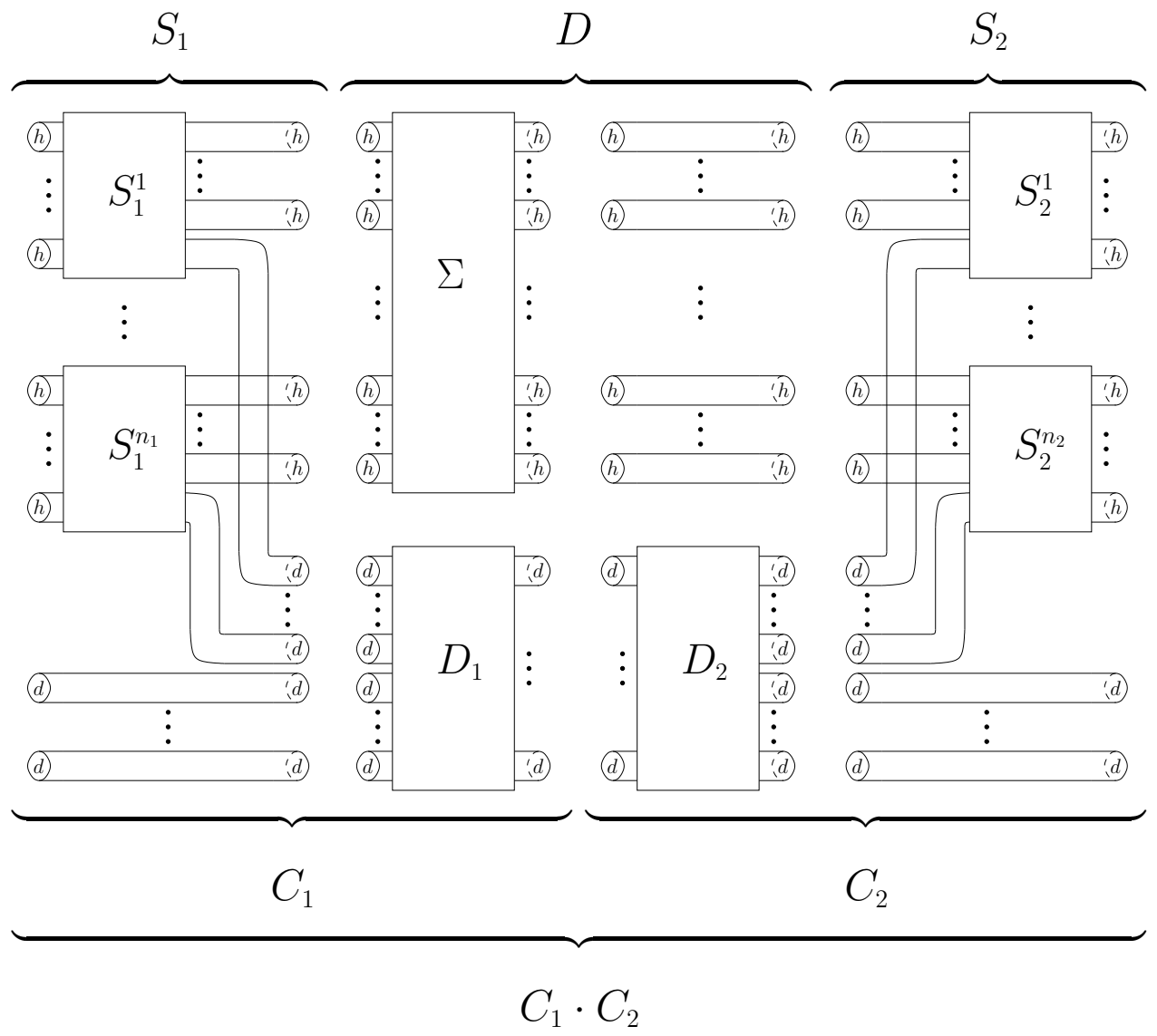

Figure 8: Cobordisms decomposition

Then, we can successively shrink all the cylinders from the upper part of $\mathcal{F}(D)$ using the relations $P R$ and $R$. We obtain a surface of genus $g$ and with $\frac{k_{1}+k_{2}}{2}$ pulleys on it where $g$ and $\left(k_{1}, k_{2}\right)$ are respectively the genus and the type of $C_{1} \cdot C_{2}$. Finally both $\mathcal{F}\left(C_{1} \cdot C_{2}\right)$ and $\mathcal{F}\left(S_{1}\right) \cdot \mathcal{F}(D) \cdot \mathcal{F}\left(S_{2}\right)$ vanish if $g+\frac{k_{1}+k_{2}}{2} \geq 2$. Otherwise, it is easily checked that the label of the possible pulley is the same in both cases.

Remark 1.8. If 2 is not assumed to be inversible in $A$, then $\mathcal{F}$ is not anymore a functor since Prop. 1.3 and its corollary do not hold. However, the relation (1) is still valid on some simple cases which are enough for our purpose.

\section{Refined Khovanov homology}

2.1. Refined geometrical Khovanov homology. Let $\left(D, \phi_{D}\right)$ be a refined diagram. Then the map $\phi_{D}$ sends naturally Bar-Natan chain complex $\llbracket D \rrbracket \in$ Kob to a chain complex $\llbracket D \rrbracket_{\phi_{D}} \in \mathrm{Kob}_{r}$. We define refined Khovanov homology as $\mathcal{F}\left(\llbracket D \rrbracket_{\phi_{D}}\right)$.

Theorem 1. The refined Khovanov homology is invariant under refined Reidemeister moves.

Proof. If 2 is invertible in $A$, then it follows from Bar-Natan results and Prop. 1.5 .

If not, then one can, fastidiously but straightforwardly, check case by case that the relation (1) holds whenever we need it to transport Bar-Natan proofs into the refined realm. 
2.2. Refined algebraic Khovanov homology. In this last section, we transport the geometrical construction given above into the algebraic world. To this end, we define some functors from $\mathcal{P}_{a b}$ to the category $A$-Mod of $A$-modules. But first, we give some notations.

Notation 2.1. Let $D$ and $H$ be the free $A$-modules generated by, respectively, $v_{+}$and $v_{-}$, and $h_{+}$ and $h_{-}$. Moreover, we denote $h_{+}+h_{-} \in H$ by $h_{0}$.

Let $m: D \otimes D \longrightarrow D, \Delta: D \longrightarrow D \otimes D, \varepsilon: A \longrightarrow D$ and $\eta: D \longrightarrow A$ be the maps defined by

$$
\begin{aligned}
& m:\left\{\begin{array}{lll}
v_{+} \otimes v_{+} & \mapsto & 0 \\
v_{ \pm} \otimes v_{\mp} & \mapsto & v_{+} \\
v_{-} \otimes v_{-} & \mapsto & v_{-}
\end{array} \quad \Delta:\left\{\begin{array}{lll}
v_{+} & \mapsto v_{+} \otimes v_{+} \\
v_{-} & \mapsto & v_{+} \otimes v_{-}+v_{-} \otimes v_{+}
\end{array}\right.\right. \\
& \varepsilon:\left\{\begin{array}{llll}
1 & \mapsto v_{-}
\end{array}:\left\{\begin{array}{lll}
v_{+} & \mapsto & 1 \\
v_{-} & \mapsto & 0
\end{array},\right.\right.
\end{aligned}
$$

and $\rho_{1}: D \longrightarrow H \otimes H, \lambda_{1}: H \otimes H \longrightarrow D, \rho_{2}: D \longrightarrow H \otimes H, \lambda_{2}: H \otimes H \longrightarrow D$ defined by

$$
\begin{aligned}
\rho_{1}:\left\{\begin{array}{lll}
v_{+} & \mapsto & 0 \\
v_{-} & \mapsto & h_{+} \otimes h_{-}+h_{-} \otimes h_{+}
\end{array}\right. & \rho_{2}:\left\{\begin{array}{lll}
v_{+} & \mapsto & 0 \\
v_{-} & \mapsto & h_{0} \otimes h_{0}+h_{-} \otimes h_{-}
\end{array}\right. \\
\lambda_{1}:\left\{\begin{array}{llll}
h_{ \pm} \otimes h_{ \pm} & \mapsto & 0 \\
h_{ \pm} \otimes h_{\mp} & \mapsto & v_{+}
\end{array}\right. & \lambda_{2}:\left\{\begin{array}{lll}
h_{+} \otimes h_{+} & \mapsto & 2 v_{+} \\
h_{ \pm} \otimes h_{\mp} & \mapsto & -v_{+} \\
h_{-} \otimes h_{-} & \mapsto & v_{+}
\end{array}\right.
\end{aligned}
$$

It is easily seen that a functor $F: \mathcal{P}_{a b} \longrightarrow A$-Mod is totally determined by its values on right and left pants, capping off and $0-$ pulleys, and on left 1 and $2-$ pulleys.

Then for all $i, j \in\{1,2\}$, we define $F_{i j}$ by $F_{i f}($ circle $)=D, F_{i f}($ dot $)=H$ and

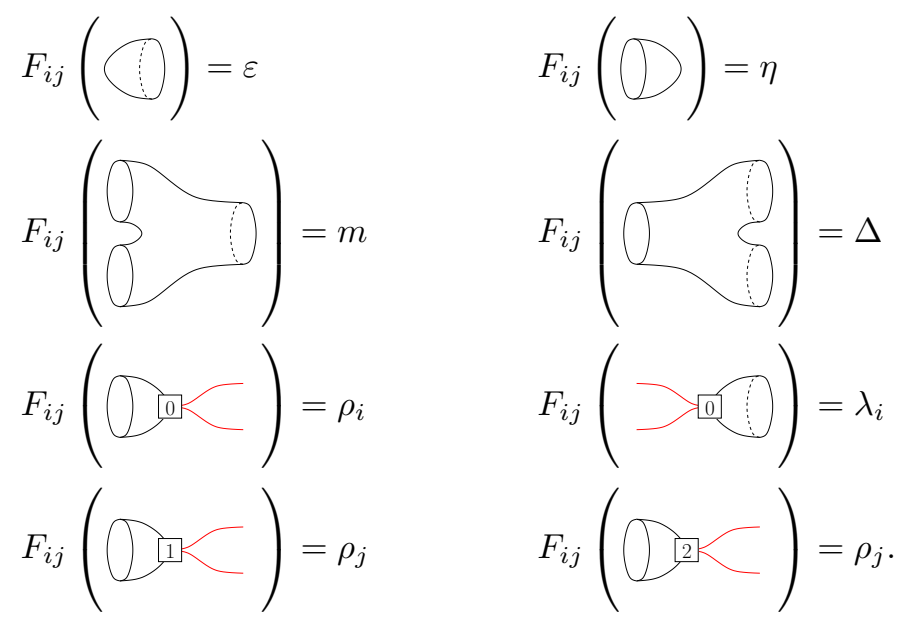

Checking that these functors are well defined, i.e. that they satisfy all relations, is left to the reader. Moreover, in the context of links in $I$-bundle, of braid-like or of star-like links, the functor $F_{11}$ send the refined geometrical Khovanov homologies to the usual refinments. 
To conclude, we give a last functor $F_{0}$ which sends pulleys with distinct labels to distinct maps. However, it requires that $A$ is of characteristic 2 .

$$
\begin{aligned}
& F_{0}(\bigcirc)=\varepsilon \\
& F_{0}(\square 0)=\left\{\begin{array}{lll}
v_{+} & \mapsto & 0 \\
v_{-} & \mapsto & h_{+} \otimes h_{+}+h_{0} \otimes h_{0}
\end{array}\right. \\
& F_{0}(\bigcirc)=\eta \\
& \left.F_{0}(\nabla 0)\right)=\left\{\begin{array}{lll}
h_{+} \otimes h_{+} & \mapsto v & v_{+} \\
h_{ \pm} \otimes h_{\mp} & \mapsto & v_{+} \\
h_{-} \otimes h_{-} & \mapsto & 0
\end{array}\right. \\
& F_{0}(O \bigcirc)=m \\
& F_{0}\left(\bigcirc 1-\left\{\begin{array}{lll}
v_{+} & \mapsto & 0 \\
v_{-} & \mapsto & h_{+} \otimes h_{+}+h_{-} \otimes h_{-}
\end{array}\right.\right. \\
& F_{0}(\Omega)=\Delta \\
& F_{0}(\square 2)=\left\{\begin{array}{lll}
v_{+} & \mapsto & 0 \\
v_{-} & \mapsto & h_{0} \otimes h_{0}+h_{-} \otimes h_{-}
\end{array} .\right.
\end{aligned}
$$

Appendix A. Relations on surfaces and Ropes

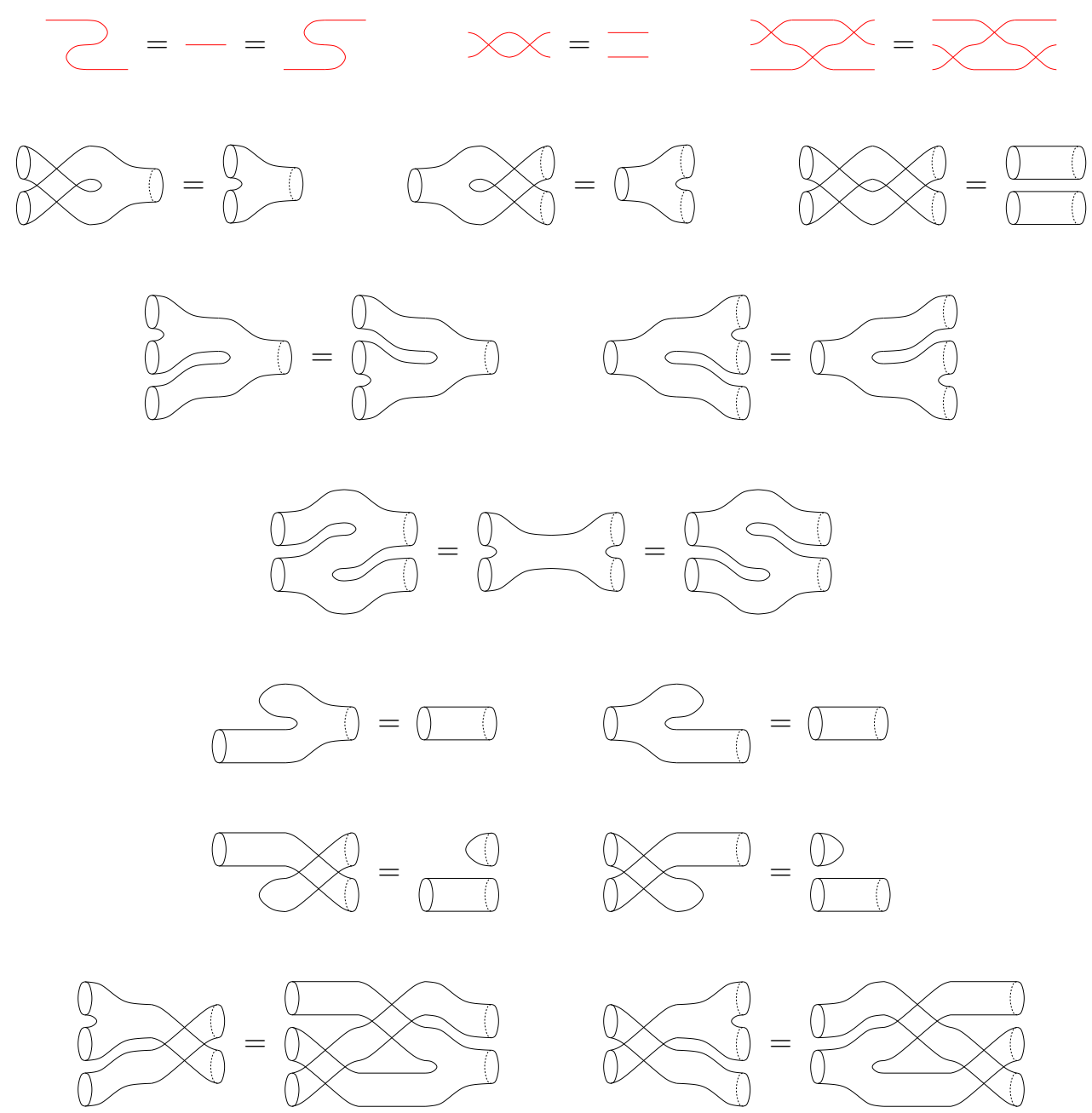




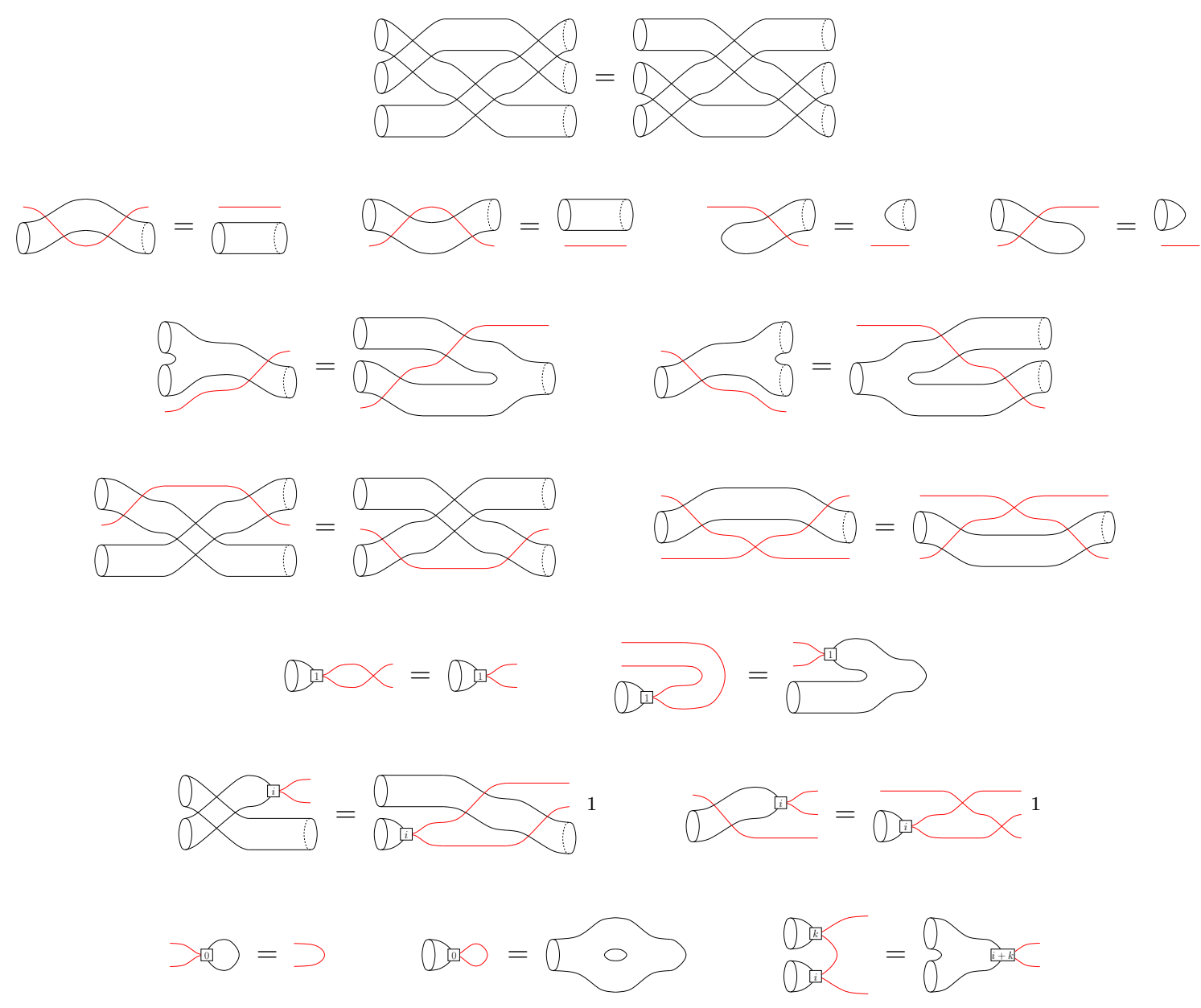

REFERENCES

[AF05] Benjamin Audoux and Thomas Fiedler, A Jones polynomial for braid-like isotopies of oriented links and its categorification, Algebr. Geom. Topol. 5 (2005), 1535-1553.

[APS04] Marta M. Asaeda, Józef H. Przytycki, and Adam S. Sikora, Categorification of the Kauffman bracket skein module of I-bundles over surfaces, Algebr. Geom. Topol. 4 (2004), 1177-1210.

[Aud09] Benjamin Audoux, Khovanov homology and star-like isotopies, Topology Appl. 156 (2009), no. 6, 10541070.

[BN05] Dror Bar-Natan, Khovanov's homology for tangles and cobordisms, Geom. Topol. 9 (2005), 1443-1499.

[CS09] J. Scott Carter and Masahico Saito, Frobenius modules and essential surface cobordisms, arXiv:math.GT/0905.4475, 2009.

[Kho00] Mikhail Khovanov, A categorification of the Jones polynomial, Duke Math. J. 101 (2000), no. 3, 359-426.

Section de Mathématiques, Unige, rue du lièvre 2-4, 1211 Genève 4, Switzerland

E-mail address: benjamin.audoux@unige.ch

\footnotetext{
${ }^{1}$ for $i=0$ and $i=1$
} 